\title{
Mobile Triage Applications: A Systematic Review in Literature and Play Store
}

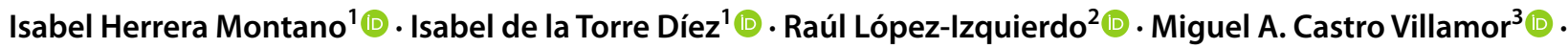 \\ Francisco Martín-Rodríguez ${ }^{3}$ (D)
}

Received: 9 April 2021 / Accepted: 5 August 2021 / Published online: 13 August 2021

(c) The Author(s), under exclusive licence to Springer Science+Business Media, LLC, part of Springer Nature 2021

\begin{abstract}
The main objective of this paper is to review and analysis of the state of the art regarding triage applications (apps) for health emergencies. This research is based on a systematic review of the literature in scientific databases from 2010 to early 2021, following a prism methodology. In addition, a Google Play Store search of the triage apps found in the literature was performed for further evaluation. A total of 26 relevant papers were obtained for this study, of which 13 apps were identified. After searching for each of these apps in the Google Play Store platform, only 2 of them were obtained, and these were subsequently evaluated together with another app obtained from the link provided in the corresponding paper. In the analysis carried out, it was detected that from 2019 onwards there has been an increase in research interest in this area, since the papers obtained from this year onwards represent $38.5 \%$ of the relevant papers. This increase may be caused by the need for early selection of the most serious patients in such difficult times for the health service. According to the review carried out, an increase in mobile app research focused on Emergency Triage and a decrease in app studies for triage catastrophe have been identified. In this study it was also observed that despite the existence of many researches in this sense, only 3 apps contained in them are accessible. "TRIAGIST" does not allow the entry of an unidentified user, "Major Trauma Triage Tool" presents negative comments from users who have used it and "ESITriage" lacks updates to improve its performance.
\end{abstract}

Keywords Triage $\cdot$ App mobile $\cdot$ Application mobile $\cdot$ Emergency Triage $\cdot$ Catastrophe Triage $\cdot$ Disaster $\cdot$ SMART . m-health

Isabel Herrera Montano

isaherrera1403@gmail.com

Isabel de la Torre Díez

isator@tel.uva.es

Raúl López-Izquierdo

rlopeziz@saludcastillayleon.es

Miguel A. Castro Villamor

mcastrovi@saludcastillayleon.es

Francisco Martín-Rodríguez

fmartin@saludcastillayleon.es

1 Department of Signal Theory and Communications and Telematics Engineering, University of Valladolid, Paseo de Belén, 15, 47011 Valladolid, Spain

2 Emergency Department. Hospital, Universitario Río Hortega, Valladolid, Spain

3 Advanced Clinical Simulation Center, School of Medicine, University of Valladolid, Valladolid, Spain

\section{Introduction}

Since the creation of the Internet, the use of devices with access to the network has grown significantly every year, generating new forms of technology in many areas of life, especially in most developed countries $[1,2]$. In the field of health and telemedicine, significant advances have been made in electronic health (e-health) or mobile health (m-health), an important development in this area being mobile health apps. These apps offer very good options for monitoring a patient's progress, as well as receiving personalized indications and support, data collection and use of self-management interventions if needed, anytime and anywhere [2].

In Spain, as in all countries of the world in the year 2020-2021, there has been a saturation of emergency 
services in each of the waves of the current COVID-19 pandemic [3-5], making it necessary to develop and optimize triage systems monitored by mobile apps, either in web environments or apps for cell phones. Many of them are also integrated by devices containing sensors to obtain information on the vital signs of patients [6].

"Triage" is the name given to the method of selection and classification of patients in prehospital care and health emergencies [7, 8], a word that comes from the French "trier". It can be defined as "the process that allows clinical risk management to adequately and safely manage patient flows when demand and clinical needs exceed resources" [2, 3].

Triage can be divided into emergency triage and catastrophe triage. Broadly speaking. Emergency triage can be identified as the "day-to-day" triage where patients arrive at the emergency department and are classified according to certain levels and, depending on their severity, the waiting time to be treated will be longer or shorter [2]. On the other hand, triage in catastrophes is when the service is faced with a catastrophe (complex emergency) and the situation becomes much more complex with a massive number of victims to be attended with insufficient resources, in this case it is necessary that the triage be fast, simple and standard in order to save the greatest number of victims with the available resources $[4,9]$.

Currently, five structured triage systems have been identified in the emergency department [2], but only two of them are used in Spain: Model Andorra de Triage (MAT) and Sistema Español de Triage (SET) [3, 6]. For emergencycatastrophe triage, the most widely used protocol is currently Simple Triage And Rapid Treatment (START) [10, 11], which was introduced in 1983 in California. This protocol classifies victims according to three vital signs: respiratory rate, heart rate and mental status. Depending on the outcome of the assessment of these vital signs, the victims are classified into four color groups indicating the victims treatment priorities [12].

This paper focuses on the review and analysis of the state of the art regarding triage apps for health emergencies. For this purpose, a description of the existing state of the art is elaborated, the results are analyzed, and the apps found are identified. Subsequently, a description of these apps is made and the most significant ones are evaluated. There are similar studies on telemedicine and triage that focus on reviewing the advances in the state of the art and the importance of symptoms for triage decisions [13], to analyze the state of use of the achievements of modern Internet technologies in connection with the tasks of screening of patients' condition by telemedicine [14], review the types of decision making processes used in intelligent healthcare systems [15], review of recent advances in mobile healthcare apps using deep learning [16] and the study of existing apps for mobile devices dedicated exclusively to the eight most prevalent health conditions according to the latest update (2004) of the Global Burden of Disease of the World Health Organization: iron deficiency anemia, hearing loss, migraine, low vision, asthma, diabetes mellitus, osteoarthritis and unipolar depressive disorders [1].

This paper presents a meta-analysis of studies focused on existing triage apps in scientific databases, including those in the Google Play Store. The frequency of publications in this regard between 2010 and early 2021 is analyzed. A total of 26 papers are analyzed in terms of their objective and origin, a total of 13 apps are identified in scientific studies and of them only 3 in Google Play Store, evaluating the latter according to medical criteria. This work is of interest for research related to the creation of new triage apps, since it provides information on existing works in scientific and academic fields, as well as those that have become accessible products for use in health emergencies.

The methodology used in this review is described in the next session. Subsequently, the results obtained are shown, the apps found in the literature are identified and the main objectives of the papers relevant to this study are analyzed. Then the results obtained are discussed and the apps found in the Google Play Store are evaluated. Finally, the conclusions obtained from this work are shown and the future lines of work that will give continuity to it are exposed.

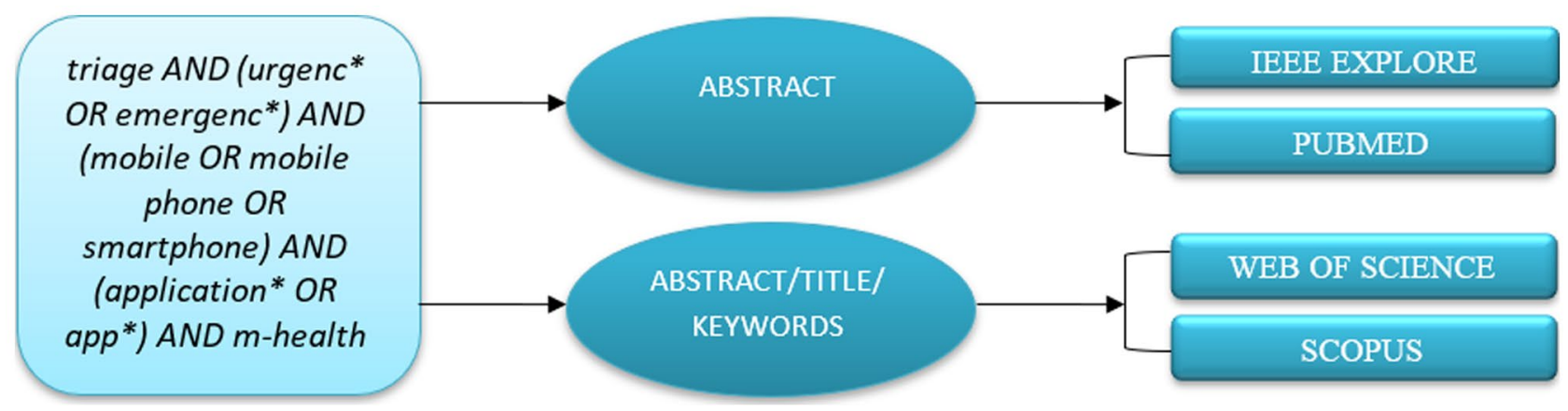

Fig. 1 Search criteria in different databases 


\section{Methodology}

This paper is based on a systematic review of the literature in scientific databases, following a prisma methodology. In addition, a search in Google Play Store of the triage apps found in the literature was performed for further evaluation. First, the scientific databases are consulted for the search and the terms used are presented, the procedure used for the selection of the articles to be analyzed and the search of the apps in Google Play Store is described.

\section{Scientific Databases}

The literature search performed in this research, focused on the scientific databases IEEE, Explore, PubMed, Web of Science and Scopus, such databases present relevant scientific content from indexed journals, repositories, archives and other text collections. The search was conducted from 2010 to 2021 using the Keywords "triage AND (urgenc* OR emergenc*) AND (mobile OR mobile phone OR smartphone) AND (application* OR app*) AND m-health" in Abstract/Title/Keywords of the papers in the databases, as shown in Fig. 1.

\section{Selection of papers}

After performing the search by entering the terms in the search engines of the databases, the articles to be analyzed were selected by reading the titles of the results obtained, resulting in 129 papers of interest in this step. Fifty-six papers that were repeated in more than one database were eliminated.

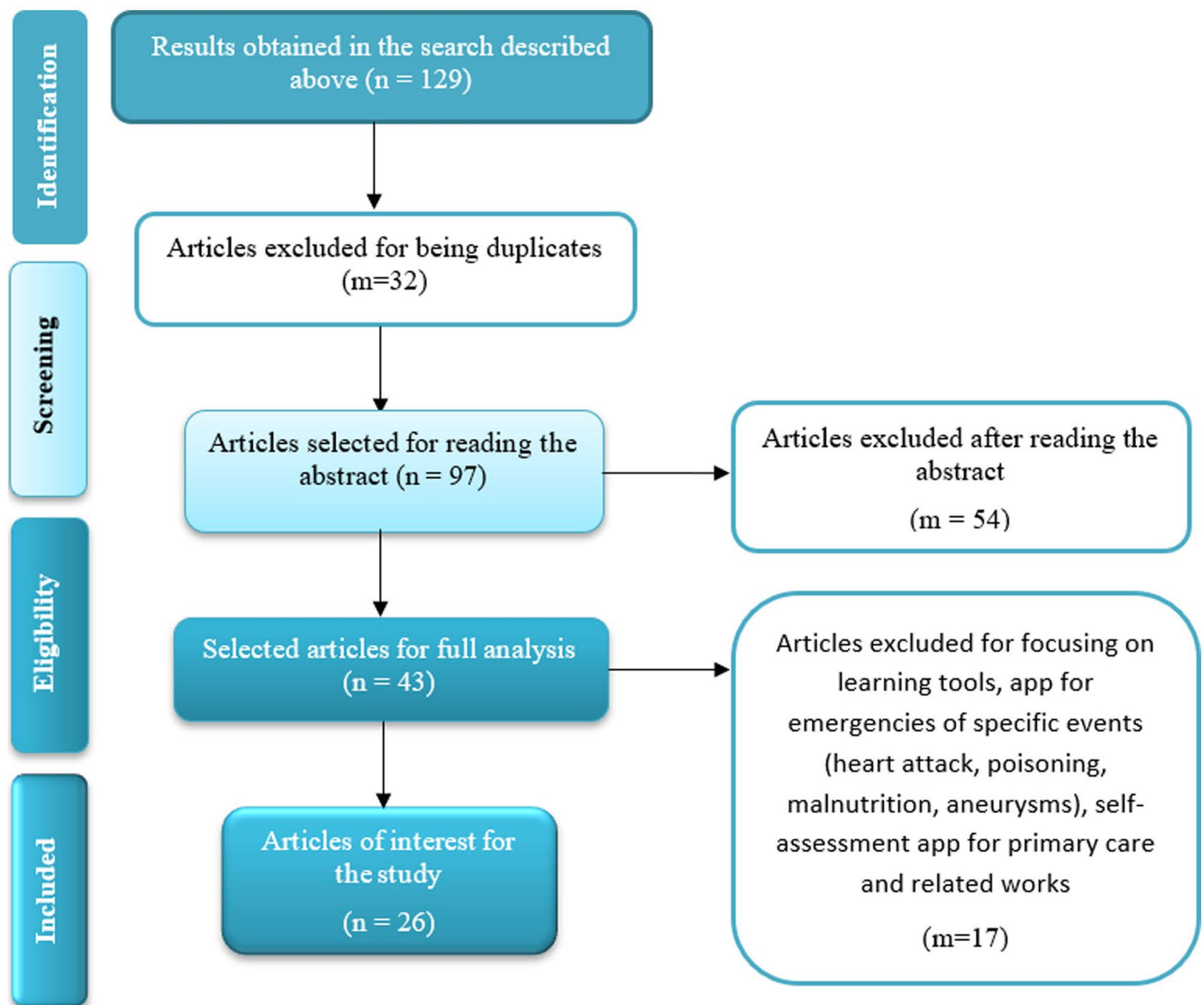

Fig. 2 Methodological Prisma for the selection of articles 
Selection criteria were applied in the analysis of the abstracts of 97 papers to classify those to be fully analyzed, the selection was focused on: 1) Studies based on novel proposals of triage apps. 2) Studies of analysis of triage apps; obtaining 43 articles for full analysis, then those studies aimed at tools to help medical students learn, emergency apps for specific events such as heart attack, poisoning, malnutrition, aneurysms, among others; self-assessment app for primary care and related works were eliminated. A total of 26 articles remained for study. This procedure is shown in the prism diagram in Fig. 2.

\section{Searching in Google Play Store}

For the evaluation of the existing triage apps in the literature, a review of the digital distribution platform of mobile apps for devices with Android operating system "Google Play" was carried out [17].

The search was performed by entering the names of the 13 apps identified in the papers of interest in the search engine of the platform. After entering the names of the apps, a total of 98 apps were obtained, of which 90 were discarded for the following reasons: 1) Irrelevance in this study because they were not apps in the medical category, 2) Theoretical-educational apps. Obtaining 8 apps that match the names of the identified apps, then the images and the description of the app are compared with the information of the paper to verify that it is the same app, from here only two of them and one that is not found on this platform but in the papers are obtained [18] is provided for easy location. The methodological PRISMA in Fig. 3 shows the methodology described.

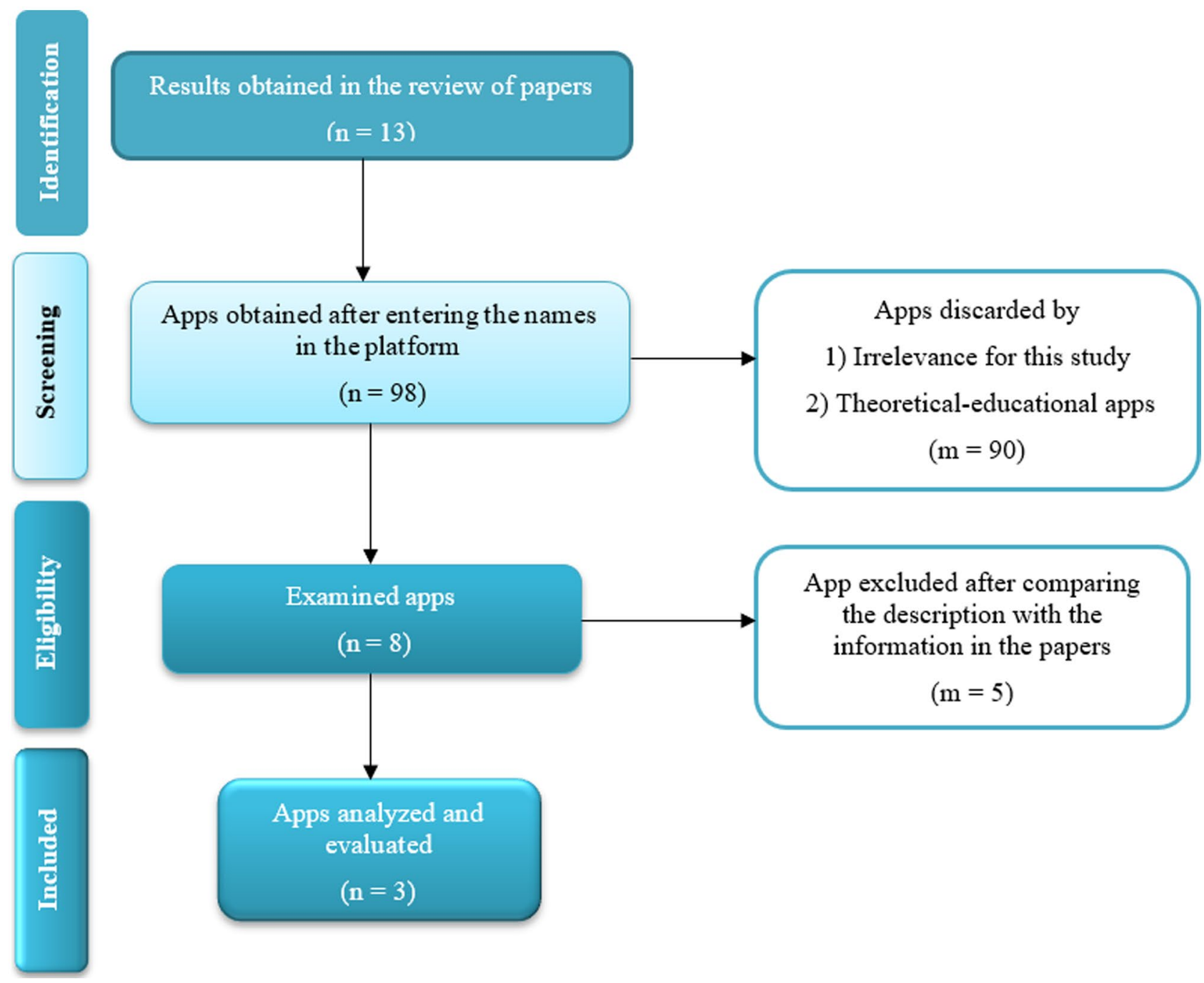

Fig. 3 Methodological Prisma for the search of apps 


\section{Results}

Once the systematic review methodology has been applied, the results obtained from the selection of relevant papers from the Google Play Store databases and apps are analyzed.

\section{Relevant Papers}

Figure 4 shows the results of relevant papers obtained in the search performed in the last 10 years, categorized according to the year of publication. It can be seen that research on this topic starts in 2012, reaching the highest number in 2020.

\section{Analysis of relevant papers}

From a total of 26 papers found 12 belong to journals, 13 are conference papers and 1 is book chapter. In the Table 1 we summarize the relevant papers that present well-defined mobile apps in terms of their name. The title, year of publication and source are shown, as well as the objective of the paper and the name of the app on which the study is based.

Other relevant papers implement apps in the proposed electronic triage systems, the following is a brief description of the study conducted in these papers.

In [12] is proposed to develop a portable medical device that can continuously monitor the health status of victims, in addition to developing an Android-based mobile app for data acquisition, priority ranking, storage and transfer of data to the medical records server in hospitals.

In [33] the authors focus on the implementation and app of a wireless patient monitoring system for triage support. This paper is a chapter from the book "WIRELESS BODY AREA NETWORKS: TECHNOLOGY,
IMPLEMENTATION, AND APPLICATIONS" which presents a discussion of similar projects, describes the design of the SMART system, and the results of its implementation in a Boston hospital.

Authors of [34] tested the feasibility of a prototype system using low-cost, commercially available components, including Radio Frequency Identification (RFID) and cell phone technology, in two simulated mass casualty incidents. On cell phones, distributed to rescue personnel at the disaster site, mTriage software is used for reading/writing RFID tags attached to "zipper collars" on victims and Logica Merlot Media Mobile software on laptops and tablets for office officers at the evacuation and treatment site.

In [35] a mobile system is developed to support emergency triage in the emergency care process for mass casualty incidents [35]. A mobile app and a data transfer mechanism are used to obtain patient data during the emergency care process. In addition, a Cox proportional hazard model is used to present the survival curve to triage service personnel as a decision aid and an injury assessment tool based on the injured patient's vital sign data.

The study conducted in [36] develops an automated triage system known as the Automated Triage System. The system consists of biomedical modules, graphical user interface (GUI) using Microsoft Visual Studio, and a triage decision making algorithm. This system acquires the patient's vital signs, syndrome and main symptoms through sensors, which are integrated into e-Health Kit V2.0, processing the data by Arduino Uno. This platform communicates and sends the acquired data to the GUI (developed for Windows) to be displayed through serial communication.

In [37] describes an optimized system designed to help the largest number of injured people in emergency

Fig. 4 Number of relevant papers for each year

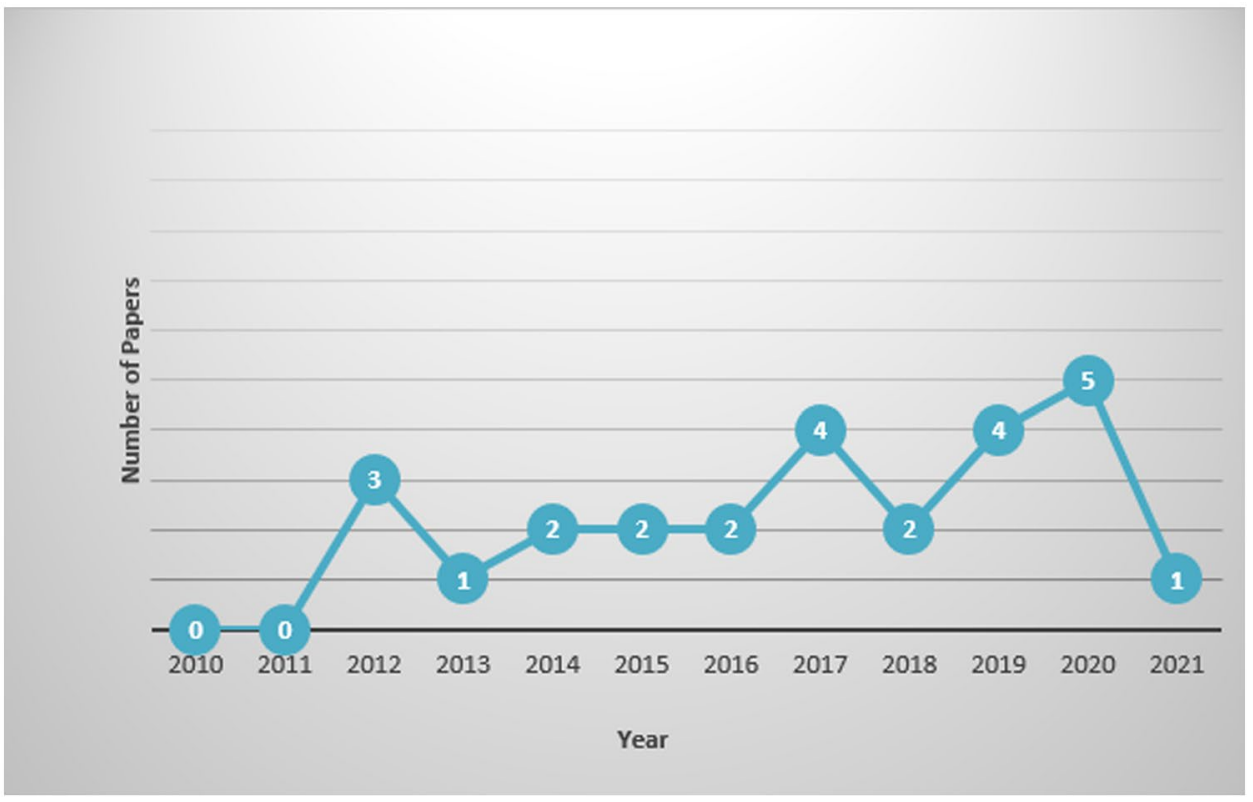




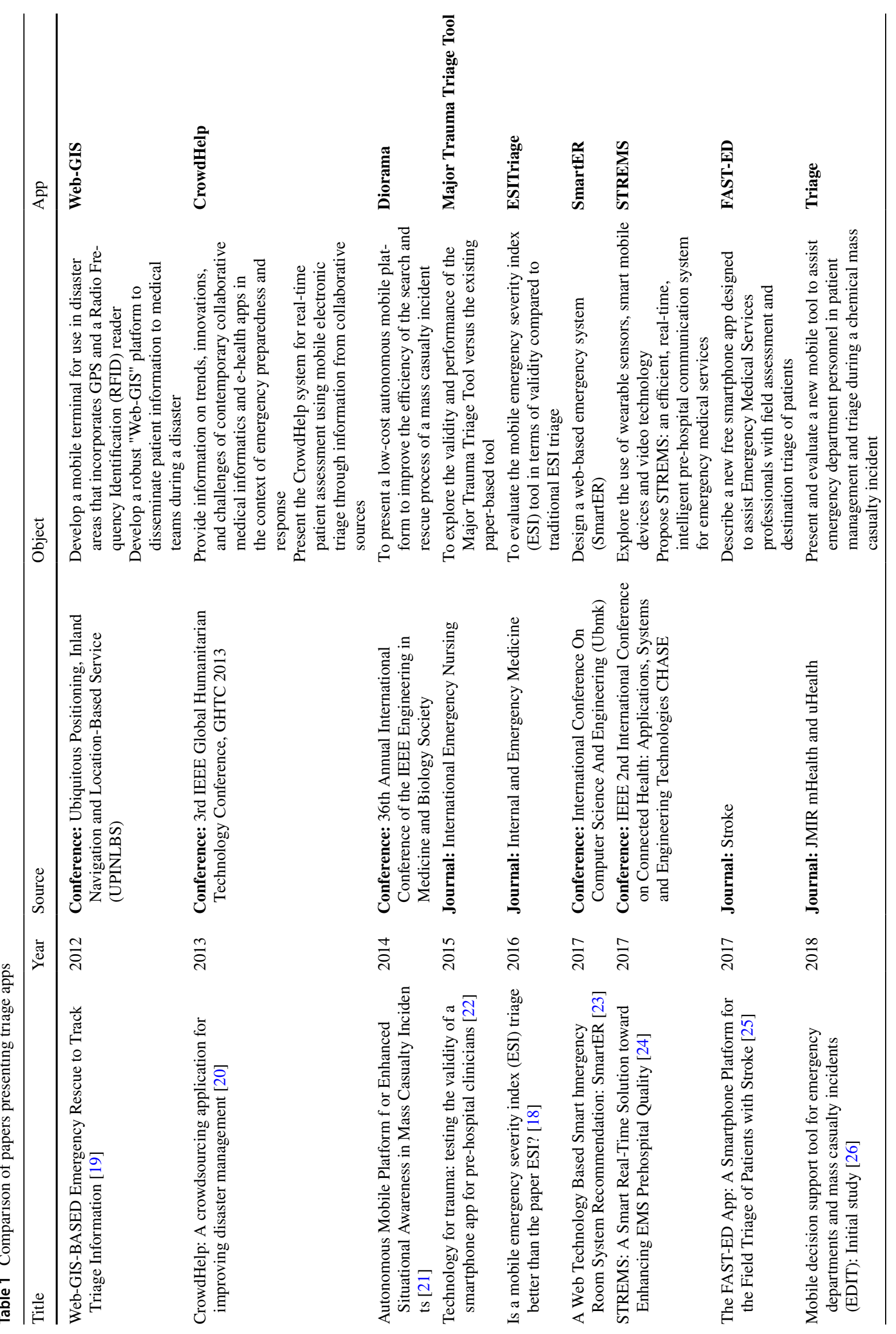




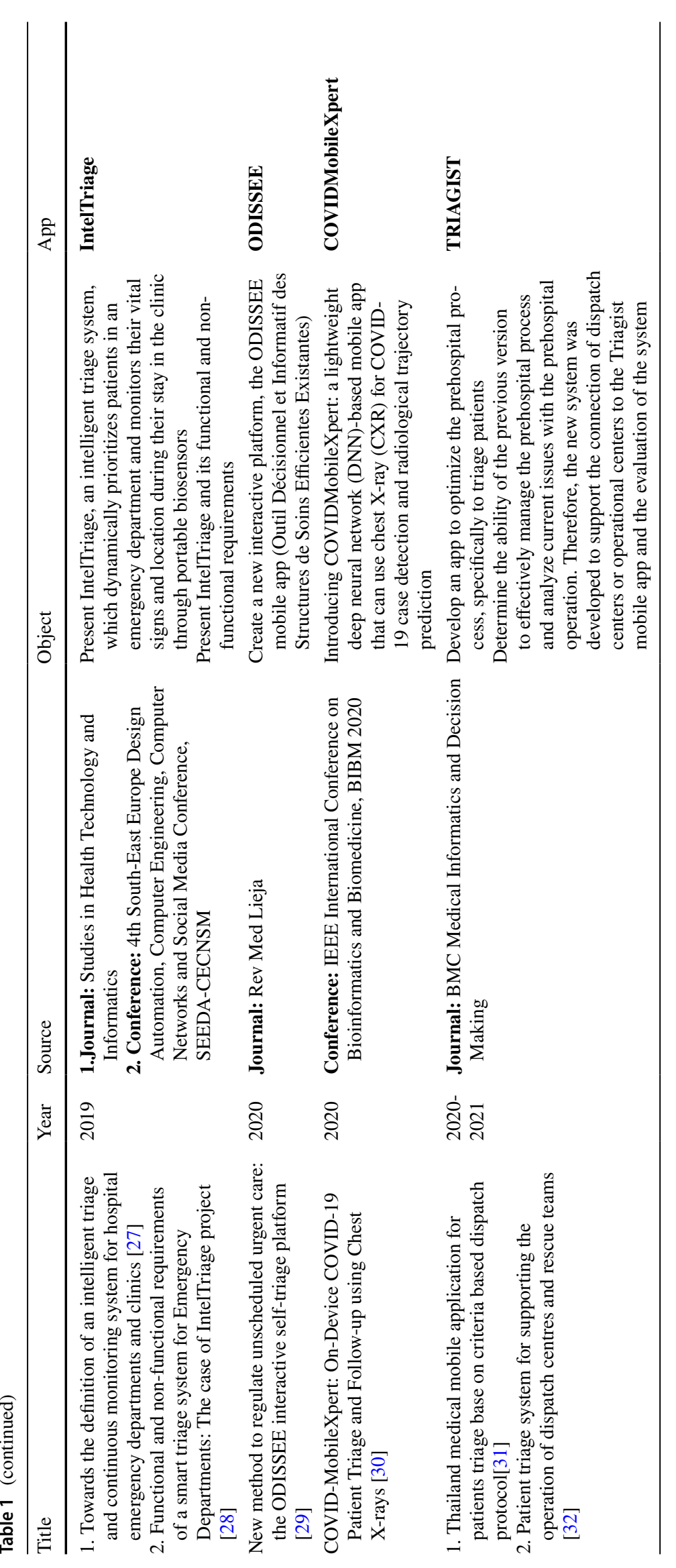


situations (IBSC). It consists of a mobile app (assigned to medical personnel and assistants), a web service and Near Field Communication wristbands assigned to the victims. The mobile app is dedicated to providing medical personnel with the geolocation of the victims, as well as an assistant that indicates the best route to follow to attend them based on the severity of their conditions and based on a triage method. Routing resolution is solved based on a classical problem, a Traveling Salesman Problem, using a $\mathrm{k}$-partition algorithm to divide the large number of victims into different groups [37].

In [38] a patient prioritization system for hospital emergency departments is presented. The proposed system consists of two main components, an innovative hospital emergency smart band (HESB) and a smart priority recommendation and patient monitoring system (SPRPC).

In [39], augmented reality was used to develop a triage algorithm and telemedicine assistance was enabled to compare the duration and quality of triage. An Android app was designed specifically for use with Smart Glasses, which added information in terms of augmented reality with two different methods: through the visualization of a triage algorithm in data glasses and a telemedical connection with a senior emergency physician performed by the integrated camera.

In [40] presents the design and implementation of a novel victim triage and identification system. The proposed solution uses Arduino software and novel electronic triage tags with enhanced capabilities, including Bluetooth connectivity, with the goal of replacing traditional paper cards.

In the paper [41] a digital triage platform is developed and implemented to rapidly identify critically ill children. It consists of a mobile app that collects clinical signs, symptoms and vital signs to prioritize children through a combination of emergency triggers and predictive risk algorithms. The results are presented on a computer-based dashboard that allows prioritization of children by displaying an overview of all and their classification categories [41].

In [42] a portable wrist device has been designed and developed that continuously monitors relevant vital signs in order to prioritize and triage Covid-19 patients in the Emergency Department. In addition, a mobile app was developed for emergency personnel for real-time detection of deteriorating health and allows highlighting the most critical Covid19 cases.

\section{Discussion}

Figure 5 shows the percentage of papers focusing on emergency triage and catastrophe triage identified in each case out of the total number of relevant papers selected. It is observed that the papers found for the years 2013-2014 and 2020-2021 base their proposals on catastrophe triage and emergency triage apps, respectively. However, in the years

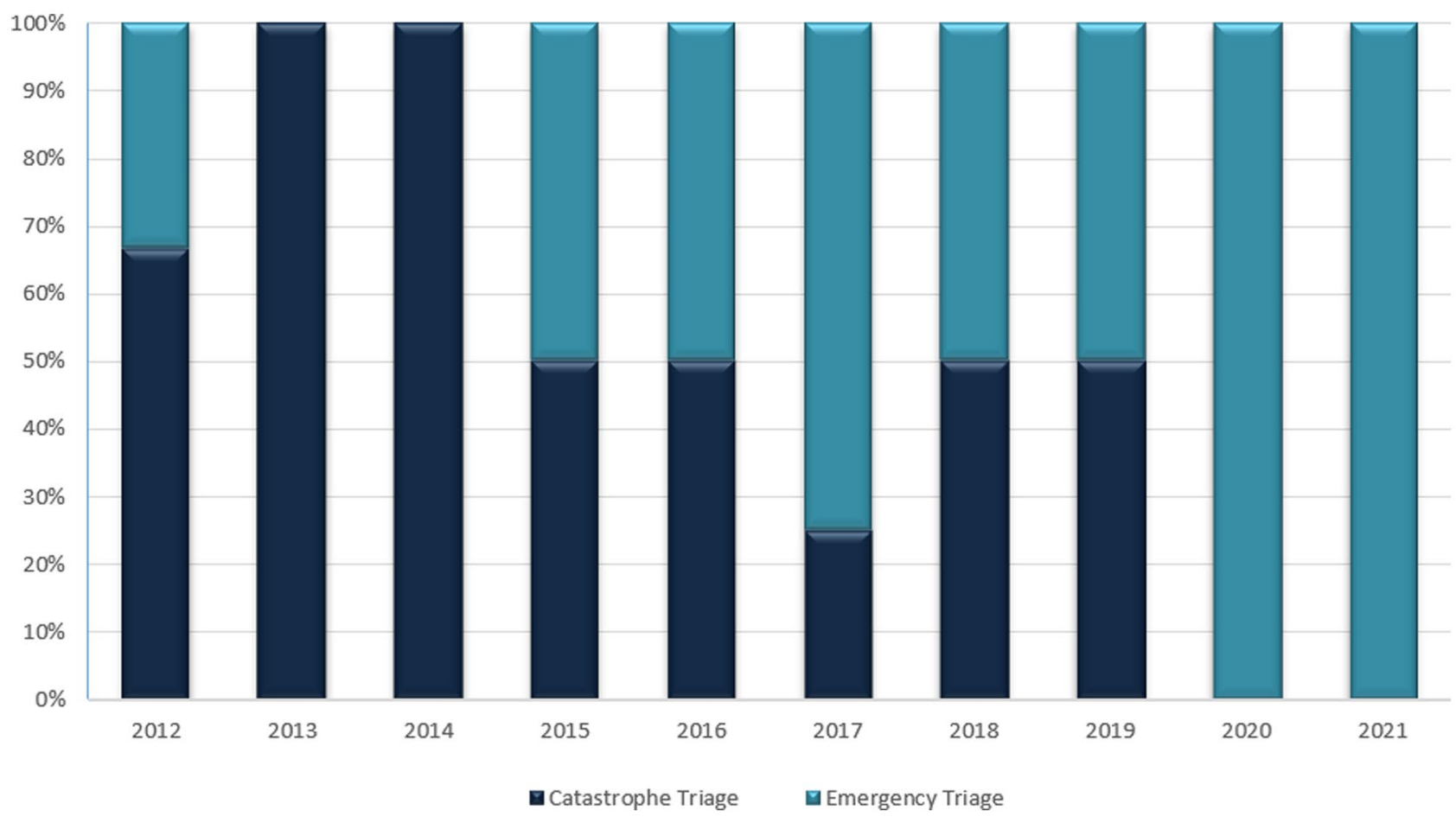

Fig. 5 Percentage of relevant papers based on catastrophe triage and emergency triage 


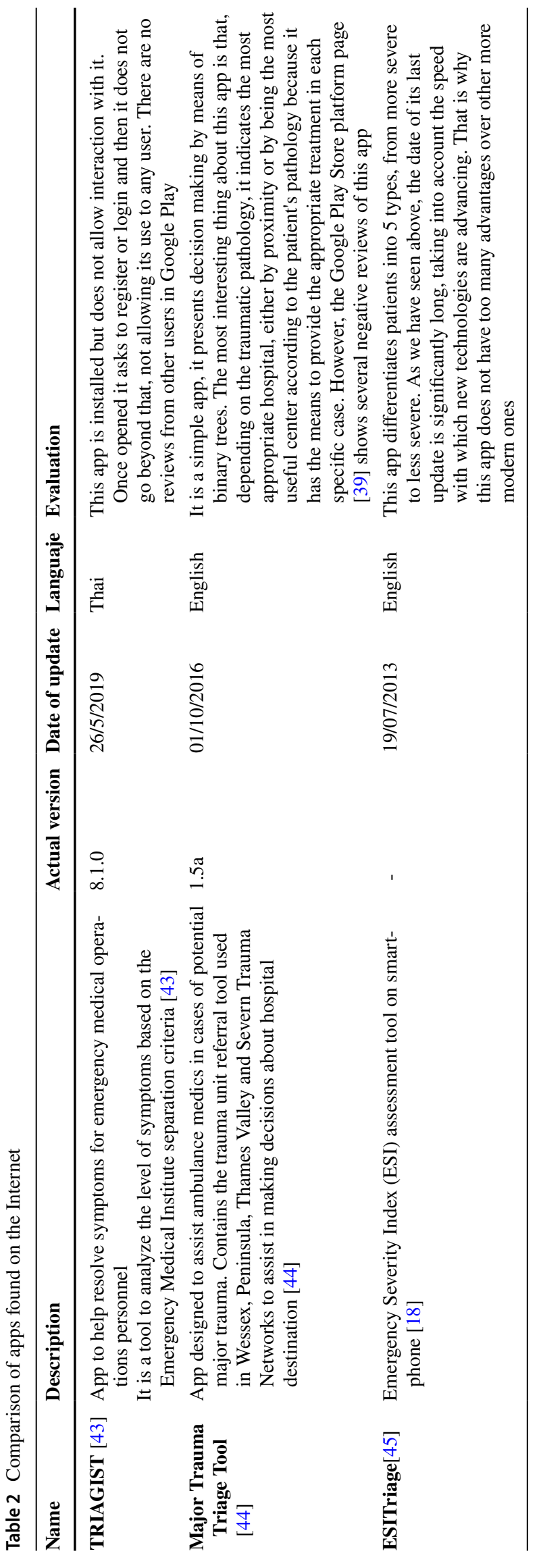

2015-2016 and 2018-2019 a balance between the objectives of the papers was identified. In 2012, almost $70 \%$ of the papers analyzed were identified as being based on catastrophe triage and in 2017 the opposite was the case. In other words, the review identified an increase in research on mobile apps focused on Emergency Triage and a decrease in studies of apps for catastrophe triage, it should be noted that most of the latter are based on the START protocol.

In the search for the apps identified in the relevant papers on the Google Play Store platform, only the "TRIAGIST" app proposed by the studies was found. [31, 32] and Major Trauma Triage Tool presented at [22].

The ESITriage app is not available on the Google Play Store platform, but in [18] the location is provided, which allows an analysis of these three apps. In this section we discuss and analyze the apps resulting from this search, downloading them and interacting with each of them to evaluate them from a clinical point of view. Table 2 shows a comparison in terms of description, current version, update date, language and evaluation.

In Table 2 it can be seen that the existing triage apps on the market, which contain a scientific and academic basis, lack free access to users external to the creating institution, or do not present continuous support for updates to incorporate the treatment into injuries caused by new pathologies, as well as other improvements to optimize the service. Therefore, this analysis reveals the need to create a new triage app that is accessible to all users, and that can also be used both in pre-hospital care and in the emergency service, providing continuous support that allows the viability of the app.

\section{Conclusions}

In this research work, a review in scientific databases of existing papers in the literature studying or proposing mobile apps for emergency triage and catastrophe triage was carried out. A total of 26 relevant papers were selected for this study, of which 13 apps were identified. After searching for each of these apps in the Google Play Store platform, only 2 of them were obtained, and these were subsequently evaluated together with another app obtained from the link provided in the corresponding paper. In the analysis carried out, it was detected that from 2019 onwards there has been increasing interest in research in this regard, given the need for early selection of the most serious patients in such difficult times for the health service. In addition, the review identified an increase in mobile app research focused on Emergency Triage and a decrease in app studies for triage catastrophe. In this study it was also observed that despite the existence of many researches in this regard only 3 apps contained in them are accessible. "TRIAGIST" does not allow the entry 
of an unidentified user, "Major Trauma Triage Tool" presents negative comments from users who have used it and "ESITriage" lacks updates to improve its performance.

In view of the above, as future lines of work, we propose the creation of an app accessible to all users that can be used both in prehospital care and in the emergency department, guaranteeing continuity of care, based on a mixed model of the physiological triage system (start) with an anatomical injury triage system. In addition, it must provide a geolocation with mapping to show the best route and the most appropriate center for the patient. It must also indicate the essential actions to be performed according to the priority level, for example: level 1, emergency (red flag), the system must ask the rescuer: have you checked the airway, is it necessary to administer oxygen, have you performed hemorrhage control, etc. This ensures the integrity of the treatment even when the rescuer is inexperienced.

Acknowledgements This research has been partially supported byEuropean Commission and the Ministry of Industry, Energy andTourism under the project AAL-20125036 named BWetake Care:ICTbased Solution for (Self-) Management of Daily Living.

\section{Compliance with Ethical Standards}

Ethical approval This article does not contain any studies with human participants or animals performed by any of the authors.

Conflict of Interest The authors declare that they have no conflict of interest.

\section{References}

1. B. Martínez-Pérez, I. De La Torre-Díez, and M. López-Coronado, "Mobile health applications for the most prevalent conditions by the world health organization: Review and analysis," J. Med. Internet Res., vol. 15, no. 6, 2013, https://doi.org/10.2196/jmir. 2600 .

2. U. Hospitalario, "Estudio del triaje en un servicio de urgencias hospitalario," vol. 5, 2013.

3. W. S. Pérez, M. Gómez Muñoz, E. Bragulat, and A. Álvarez, "Triage: A key tool in emergency care," An. Sist. Sanit. Navar., vol. 33, no. SUPP1, pp. 55-68, 2010.

4. S. R. Macho, "Triage en emergencias y catástrofes extrahospitalarias. Nuevo reto para la enfermería," Nuberos Cient., vol. 3, no. 25 , pp. 67-73, 2018

5. D. M. Morens and A. S. Fauci, "EmergingPandemicDiseases:How WeGottoCOVID-19," Cell, vol. 182, no. January, pp. 1077-1092, 2020.

6. J. Gómez Jiménez, F. Boneu Olaya, O. Becerra Cremidis, E. Albert Cortés, J. Ferrando Garrigós, and M. MEdina Prats, "Validación clínica de la nueva versión del Programa de Ayuda al Triaje (web_ e-PAT v3) del Modelo Andorrano de Triaje (MAT) y Sistema Español de Triaje (SET). Fiabilidad, utilidad y validez en la población pediátrica y adulta," Emergencias Rev. la Soc. Española Med. Urgencias y Emergencias, vol. 18, no. 4, pp. 207-214, 2006.

7. E. Medina-Lozano, F. Martín-Rodríguez, M. Castro-Villamor, C. Escudero-Cuadrillero, C. del P. Vegas, and R. López-Izquierdo,
"Accuracy of early warning scores for predicting serious adverse events in pre-hospital traumatic injury," Injury, vol. 51, no. 7, pp. 1554-1560, 2020, https://doi.org/10.1016/j.injury.2020.04.042.

8. F. Martín-Rodríguez et al., "Accuracy of National Early Warning Score 2 (NEWS2) in Prehospital Triage on In-Hospital Early Mortality: A Multi-Center Observational Prospective Cohort Study," Prehosp. Disaster Med., vol. 34, no. 6, pp. 610-618, 2019, https://doi.org/10.1017/S1049023X19005041.

9. M. Pepper, F. Archer, and J. Moloney, "Triage in Complex, Coordinated Terrorist Attacks," Prehosp. Disaster Med., vol. 34, no. 4, pp. 442-448, 2019, doi: https://doi.org/10.1017/ S1049023X1900459X.

10. M. E. Demirel, İ. H. Ali, and M. Boğan, "Emergency service experience following the terrorist attack in Mogadishu, 14 October 2017, a scene of lay rescuer triage," Am. J. Emerg. Med., vol. 40, pp. 6-10, 2021, https://doi.org/10.1016/j.ajem.2020.12. 005 .

11. A. A. Shah et al., "Impact of a predefined hospital mass casualty response plan in a limited resource setting with no pre-hospital care system," Injury, vol. 46, no. 1, pp. 156-161, 2015, doi: https://doi.org/10.1016/j.injury.2014.08.029.

12. M. Niswar et al., "The design of wearable medical device for triaging disaster casualties in developing countries," in 2015 5th International Conference on Digital Information Processing and Communications, ICDIPC 2015, 2015, pp. 207-212, https://doi. org/10.1109/ICDIPC.2015.7323030.

13. B. Roland and H. Nickel Christian, "The last decade of symptomoriented research in emergency medicine: Triage, work-up, and disposition," Swiss Med. Wkly., vol. 149, no. 41-42, pp. 2-9, 2019,https://doi.org/10.4414/smw.2019.20141.

14. K. Kolisnyk, D. Deineko, T. Sokol, S. Kutsevlyak, and O. Avrunin, "Application of modern internet technologies in telemedicine screening of patient conditions," 2019 IEEE Int. Sci. Conf. Probl. Infocommunications Sci. Technol. PIC S T 2019 - Proc., pp. 459464, 2019, https://doi.org/10.1109/PICST47496.2019.9061252.

15. Q. Cai, H. Wang, Z. Li, and X. Liu, "A Survey on Multimodal Data-Driven Smart Healthcare Systems: Approaches and Applications," IEEE Access, vol. 7, pp. 133583-133599, 2019, https:// doi.org/10.1109/ACCESS.2019.2941419.

16. G. N. Hd et al., "An Initial Framework for Movile Healthcare Systems using Deep Neural Networks," 2019, pp. 205-210.

17. S. McIlroy, N. Ali, and A. E. Hassan, "Fresh apps: an empirical study of frequently-updated mobile apps in the Google play store," Empir. Softw. Eng., vol. 21, no. 3, pp. 1346-1370, 2016, https:// doi.org/10.1007/s10664-015-9388-2.

18. S. Savatmongkorngul, C. Yuksen, C. Suwattanasilp, K. Sawanyawisuth, and Y. Sittichanbuncha, "Is a mobile emergency severity index (ESI) triage better than the paper ESI?," Intern. Emerg. Med., vol. 12, no. 8, pp. 1273-1277, 2016, https://doi.org/ 10.1007/s11739-016-1572-x.

19. K. Komatsu, H. Imai, and K. Nishimori, "Web-GIS-BASED Emergency Rescue to Track Triage Information," in 2012 Ubiquitous Positioning, Inland Navigation and Location-Based Service (UPINLBS), 2012, pp. 1-4.

20. L. I. Besaleva and A. C. Weaver, "CrowdHelp: A crowdsourcing application for improving disaster management," in Proceedings of the $3 \mathrm{rd}$ IEEE Global Humanitarian Technology Conference, GHTC 2013, 2013, pp. 185-190, https://doi.org/10.1109/GHTC.2013.6713678.

21. D. Yang, J. Schafer, S. Wang, and A. Ganz, "Autonomous Mobile Platform $\mathrm{f}$ or Enhanced Situational Awareness in Mass Casualty Inciden ts," in 36th Annual International Conference of the IEEE Engineering in Medicine and Biology Society, 2014, pp. 898-901.

22. E. S. Freshwater and R. Crouch, "Technology for trauma: Testing the validity of a smartphone app for pre-hospital clinicians," Int. Emerg. Nurs., vol. 23, no. 1, pp. 32-37, 2015, https://doi.org/10. 1016/j.ienj.2014.04.003. 
23. C. Toremis and E. Karaarslan, "A Web Technology Based Smart hmergency Room System Recommendation: SmartER," in INTERNATIONAL CONFERENCE ON COMPUTER SCIENCE AND ENGINEERING (UBMK), 2017, pp. 5-9.

24. X. Wu, R. Dunne, Z. Yu, and W. Shi, "STREMS: A Smart RealTime Solution toward Enhancing EMS Prehospital Quality," in Proceedings - 2017 IEEE 2nd International Conference on Connected Health: Applications, Systems and Engineering Technologies, CHASE 2017, 2017, pp. 365-372, https://doi.org/10.1109/ CHASE.2017.120.

25. R. G. Nogueira et al., "The FAST-ED App: A Smartphone Platform for the Field Triage of Patients with Stroke," Stroke, vol. 48, no. 5, pp. 1278-1284, 2017, https://doi.org/10.1161/STROKEAHA.116. 016026.

26. N. Boltin, D. Valdes, J. M. Culley, and H. Valafar, "Mobile decision support tool for emergency departments and mass casualty incidents (EDIT): Initial study," JMIR mHealth uHealth, vol. 6, no. 6, pp. 1-15, 2018, https://doi.org/10.2196/10727.

27. A. Billis et al., "Towards the definition of an intelligent triage and continuous monitoring system for hospital emergency departments and clinics," Stud. Health Technol. Inform., vol. 264, pp. 1641-1642, 2019, https://doi.org/10.3233/SHTI190574.

28. A. Billis et al., "Functional and non-functional requirements of a smart triage system for Emergency Departments: The case of IntelTriage project," in 2019 4th South-East Europe Design Automation, Computer Engineering, Computer Networks and Social Media Conference, SEEDA-CECNSM 2019, 2019, pp. 20192022, https://doi.org/10.1109/SEEDA-CECNSM.2019.8908320.

29. D. V Gilbert A, brAsseur e, Ghuysen A, "New method to regulate unscheduled urgent care : the ODISSEE interactive self-triage platform," Rev Med Lieja., vol. 75 (3), no. 1, pp. 159-163, 2020.

30. X. Li, C. Li, and D. Zhu, "COVID-MobileXpert: On-Device COVID-19 Patient Triage and Follow-up using Chest X-rays," in Proceedings - 2020 IEEE International Conference on Bioinformatics and Biomedicine, BIBM 2020, 2020, pp. 1063-1067, https://doi.org/10.1109/BIBM49941.2020.9313217.

31. K. Sutham, P. Khuwuthyakorn, and O. Thinnukool, "Thailand medical mobile application for patients triage base on criteria based dispatch protocol," BMC Med. Inform. Decis. Mak., vol. 20, no. 1, pp. 1-13, 2020, https://doi.org/10.1186/s12911-020-1075-6.

32. A. Nimmolrat, K. Sutham, and O. Thinnukool, "Patient triage system for supporting the operation of dispatch centres and rescue teams," BMC Med. Inform. Decis. Mak., vol. 21, no. 1, pp. 1-16, 2021, https://doi.org/10.1186/s12911-021-01440-x.

33. L Pino EJ (Pino, Esteban J.)[1 ]; Curtis, D (Curtis, Dorothy); Stair, TO (Stair, Tom O.); Guttag, JV (Guttag, John V.); OhnoMachado, L (Ohno-Machado, "Wireless Patient Monitoring in a Clinical Setting", in WIRELESS BODY AREA NETWORKS: TECHNOLOGY, IMPLEMENTATION, AND APPLICATIONS, Pinoa, E. J., Curtisb, D., Stairc, T. O., Guttagb, J. V., \& Ohno-Machadod, L. 2011 Wireless Patient Monitoring in a Clinical Setting Wireless Body Area Networks: Technology, Implementation, and Applications 1920121940
34. J. Jokela et al., "Increased situation awareness in major incidents'radio frequency identification (RFID) Technique: A promising tool," Prehosp. Disaster Med., vol. 27, no. 1, pp. 81-87, 2012, https://doi.org/10.1017/S1049023X12000295.

35. Y. Tian, T. S. Zhou, Y. Wang, M. Zhang, and J. S. Li, "Design and development of a mobile-based system for supporting emergency triage decision making mobile systems," J. Med. Syst., vol. 38, no. 6, 2014, https://doi.org/10.1007/s10916-014-0065-6.

36. H. A. Chong and K. B. Gan, "Development of automated triage system for emergency medical service," in 2016 International Conference on Advances in Electrical, Electronic and Systems Engineering, ICAEES 2016, 2016, pp. 642-645, https://doi.org/ 10.1109/ICAEES.2016.7888125.

37. A. Rivero-García, I. Santos-González, C. Hernández-Goya, and P. Caballero-Gil, "IBSC system for victims management in emergency scenarios," in IoTBDS 2017 - Proceedings of the 2nd International Conference on Internet of Things, Big Data and Security, 2017, no. IoTBDS, pp. 276-283, https://doi.org/10.5220/0006298702760283.

38. B. Lima and J. P. Faria, "Towards real-time patient prioritization in hospital emergency services," 2018, https://doi.org/10.1109/ HealthCom.2018.8531089.

39. A. Follmann, M. Ohligs, N. Hochhausen, S. K. Beckers, R. Rossaint, and M. Czaplik, "Technical support by smart glasses during a mass casualty incident: A randomized controlled simulation trial on technically assisted triage and telemedical app use in disaster medicine," J. Med. Internet Res., vol. 21, no. 1, pp. 1-10, 2019, https://doi.org/10.2196/11939.

40. A. Rigos et al., "A novel triage system using Bluetooth devices in support of incident management," in Proceedings of the International Conference on Sensing Technology, ICST, 2019, vol. 2019Decem, no. c, https://doi.org/10.1109/ICST46873.2019.9047744.

41. V. Lee et al., "Evaluation of a digital triage platform in Uganda: A quality improvement initiative to reduce the time to antibiotic administration," PLoS One, vol. 15, no. 10 October, pp. 1-15, 2020, https://doi.org/10.1371/journal.pone.0240092.

42. B. Fyntanidou et al., "IoT-based smart triage of Covid-19 suspicious cases in the Emergency Department," in 2020 IEEE Global Communications Conference (GLOBECOM), 2020, no. 2020, pp. $8-13$.

43. Orawit.t@gmail.com, “TRIAGIST,” Google Play, 2019. https:// play.google.com/store/apps/details?id=th.ac.cmu.camt.triage.

44. Contact@ volatilestate.com, "Major Trauma Triage Tool," Google Play, 2016. https://play.google.com/store/apps/details?id=com. volatilestate.triage \&hl=en_IN.

45. S. Savatmongkorngul, C. Yuksen, C. Suwattanasilp, K. Sawanyawisuth, and Y. Sittichanbuncha, "ESITriage.apk," mediafire, 2013. https:// www.mediafire.com/file/dbk9ixqbwtxgbm2/ESITriage.apk/file.

Publisher's Note Springer Nature remains neutral with regard to jurisdictional claims in published maps and institutional affiliations. 